\title{
A study on effect of social capital on expediting knowledge distribution
}

\author{
Habibollah Javanmard ${ }^{\mathrm{a}}$ and Zinat Sadat Alhosseini ${ }^{\mathrm{b}^{*}}$
}

${ }^{a}$ Assistant Professor, Arak Branch, Islamic Azad University, Arak, Iran

${ }^{b}$ Masters Student, Arak Branch, Islamic Azad University, Arak, Iran

\section{CHR ON I C L E A B S T RACT}

Article history:

Received May 16, 2013

Received in revised format

18 August 2013

Accepted 18 August 2013

Available online

August $28 \quad 2013$

Keywords:

Social capital

Knowledge distribution

Knowledge management

\begin{abstract}
Knowledge plays important role on having continuous improvement within organization. Highly skilled employees normally contribute their knowledge within organizations and help others. In this paper, we perform an empirical investigation to find important factors influencing expediting knowledge distribution in one of Iranian organizations. The proposed study of this paper designs a questionnaire and distributes it among a randomly selected employees and using some statistical test, the relationship between knowledge distribution as dependent variables with eight independent variables are investigated. The results of our survey confirm that informal mechanism, building a good trust within organization, having a good interaction among different units of organization, hiring highly committed employees, improving innovation, and learning capabilities within organization may help expedite distribution of knowledge within organization. However, the survey does not find any statistical evidence to believe that formal mechanism, having a good identity and stating objective had any meaningful impact on distributing knowledge within organization.
\end{abstract}

\section{Introduction}

A healthy organization normally is able to provide good infrastructure to share employees' capabilities within different units. Sharing knowledge helps organizations grow faster and facilitate any possibilities for creativity and innovation (Wang \& Noe, 2010). There are, however, some concerns on how we can make knowledge distribution faster and more reliable. Jung (2009), for instance, proposed an architecture to fulfill context matching among blog-based knowledge management systems referred to as detecting 'shared' context between knowledge management systems. Thus, they wish to detect a community of practice $(\mathrm{CoP})$ on a knowledge blogosphere where 
a set of blog-based knowledge management systems were incorporating with each other. As a result, newly generated knowledge could be proactively diffused to the blog-based knowledge management systems in which context was relevant to the knowledge, even before the bloggers' queries were explicitly requested.

Berglund (2005) described design criteria for bug handing from a global user community perspective. The author described a distribution architecture for bug knowledge and concentrated on bug awareness and bug visibility in the standard work environment. According to Castellacci and Archibugi (2008), the convergence clubs literature in applied growth theory implies that countries that differ in terms of structural characteristics and initial conditions will more likely experience diverging growth performances. They investigated this unexplored question by carrying out a study of the cross-country distribution of knowledge in a large sample of developed and developing economies in the 1990s. They indicated the existence of three technology clubs characterized by markedly various levels of development. The clubs also differed with respect to the dynamics of their capabilities over the decade, as the most advanced group and the intermediate one were detected to be much more dynamic than the large cluster of less developed economies. Nussbaum et al. (1997) reported the experience of solving the distribution problem for the biggest fuel company in Chile.

Liu et al. (2011) discussed the challenges of knowledge sharing (KS) in dynamic virtual enterprises. They introduced a KS community model and adopted an agent based solution to execute the functions of KS among members of a dynamic virtual enterprise. The method incorporated the concepts of agent society and semantic ontology where KS in dynamic virtual enterprises was performed with three kinds of ties: knowledge agent to knowledge agent, knowledge agent to knowledge item, and knowledge item to knowledge item. They measured agent-to-agent ties by preference correlation implementing the contribution degree of one agent to another and the preference similarity degree between two agents. They defined a semantic view to demonstrate agent-to-item ties and applied semantic links in the knowledge resource space model to reflect item-to-item ties. They also elaborated the co-evolution mechanism of collective intelligence across enterprises throughout the lifecycle of a dynamic virtual enterprise.

\section{The proposed model}

The proposed model of this paper investigates whether social capital could help expedite the process of knowledge distribution. Fig. 1 demonstrates the proposed study of this paper.

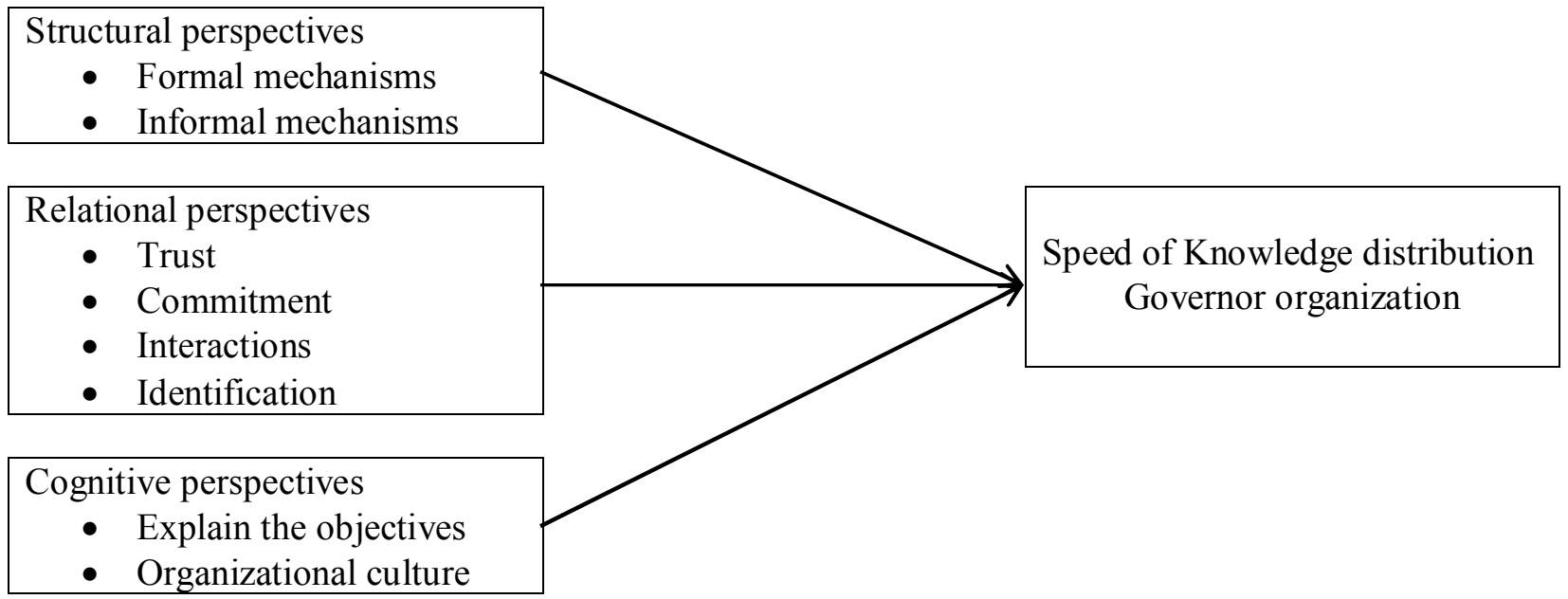

Fig. 1. The proposed study 
According to Fig. 1, we consider the following eight sub-hypotheses,

1. Formal mechanisms within organization facilities distribution of knowledge.

2. Informal mechanisms within organization facilities distribution of knowledge.

3. Trust in receiver of knowledge facilitates distribution of knowledge.

4. Having a good interactions between different units expedite the process of distrusting knowledge.

5. Existence of trust between different units expedites the process of distrusting knowledge.

6. Having a good identification among different units expedite the process of distrusting knowledge.

7. Stating precise objectives and accepting them within organization help expedite the process of distrusting knowledge.

8. A high level of organizational culture with innovation and learning capabilities help distribution of knowledge within organization.

The above eight factors have already been investigated in other similar studies (e.g. Ipe, M., 2003; Jones et al., 2006; Sánchez et al., 2013).

The proposed study has been accomplished in different units affiliated with governor organization in province of Markazin, Iran. We have determined that there were 190 employees working as engineer or managers. Therefore, the sample size is calculated as follows,

$$
n=\frac{N \times z_{\alpha / 2}^{2} \times p \times q}{\varepsilon^{2} \times(N-1)+z_{\alpha / 2}^{2} \times p \times q},
$$

where $N$ is the population size, $p=1-q$ represents the yes/no categories, $z_{\alpha / 2}$ is CDF of normal distribution and finally $\varepsilon$ is the error term. Since we have $p=0.5, z_{\alpha / 2}=1.96$ and $N=190$, the number of sample size is calculated as $n=130$. The proposed study uses regression analysis to verify the relationship between different components of the survey where a faster rate of knowledge distribution is the dependent variable and the components shown in Fig. 1 are independent variables.

\section{The results}

In this section, we present details of our findings on testing eight hypotheses of this survey. Table 1 summarizes the results.

Table 1

The results of the relationship between different organizational factors with knowledge distribution

\begin{tabular}{llccccc}
\hline Independent variable & \multicolumn{1}{l}{ Non-standard coefficients } & Standard coefficient & t-value & Sig. & Result \\
\hline Formal mechanism & 0.009 & 0.093 & 0.008 & 0.098 & 0.992 & $\times$ \\
Informal mechanism & 0.218 & 0.099 & 0.222 & 2.205 & 0.029 & $\sqrt{ }$ \\
Trust & 0.198 & 0.076 & 0.191 & 2.601 & 0.011 & $\sqrt{ }$ \\
Interactions & 0.210 & 0.102 & 0.212 & 2.051 & 0.042 & $\sqrt{ }$ \\
Commitment & 0.160 & 0.079 & 0.143 & 2.024 & 0.045 & $\sqrt{ }$ \\
Identity & 0.002 & 0.083 & 0.002 & 0.019 & 0.984 & $\times$ \\
Stating objectives & 0.018 & 0.082 & 0.018 & 0.222 & 0.825 & $\times$ \\
Organizational culture & 0.210 & 0.105 & 0.193 & 2.007 & 0.047 & $\sqrt{ }$ \\
\hline
\end{tabular}


The results of Table 1 indicate that five independent variables including "Informal mechanism", "Trust", "Interactions", "Commitment" and "Organizational culture" influence on knowledge distribution, significantly $(\alpha=5 \%)$. However, formal mechanism, having a good identity and stating objective do not have meaningful impact on distributing knowledge within organization.

\section{Conclusion}

In this paper, we have presented an empirical investigation to study the impact of eight variables on expediting knowledge distribution in one of Iranian governmental organization. The proposed study used regression analysis where the dependent variable was distribution of knowledge and eight variables were considered as independent variable in eight regression models. The results of our survey have confirmed that informal mechanism, building a good trust within organization, having a good interaction among different units of organization, hiring highly committed employees, improving innovation, and learning capabilities within organization may help expedite distribution of knowledge within organization. However, the survey did not find any statistical evidence to believe that formal mechanism, having a good identity and stating objective had any meaningful impact on distributing knowledge within organization.

\section{Acknowledgement}

The authors would like to thank the anonymous referees for constructive comments on earlier version of this paper.

\section{References}

Berglund, E. (2005). Communicating bugs: global bug knowledge distribution. Information and Software Technology, 47(11), 709-719.

Castellacci, F., \& Archibugi, D. (2008). The technology clubs: The distribution of knowledge across nations. Research Policy, 37(10), 1659-1673.

Jones, M. C., Cline, M., \& Ryan, S. (2006). Exploring knowledge sharing in ERP implementation: an organizational culture framework. Decision Support Systems, 41(2), 411-434.

Jung, J.J. (2009). Knowledge distribution via shared context between blog-based knowledge management systems: A case study of collaborative tagging. Expert Systems with Applications, 36(7), 10627-10633.

Liu, P., Raahemi, B., Benyoucef, M. (2011). Knowledge sharing in dynamic virtual enterprises: A socio-technological perspective. Knowledge-Based Systems, 24(3), 427-443.

Ipe, M. (2003). Knowledge sharing in organizations: a conceptual framework. Human Resource Development Review, 2(4), 337-359.

Nussbaum, M. Sepulveda, M., Cobian, A., Gaete, J., Parra, E., \& Cruz, J. (1997). A fuel distribution knowledge-based decision support system. Omega, 25(2), 225-234.

Sánchez, J. H., Sánchez, Y. H., Collado-Ruiz, D., \& Cebrián-Tarrasón, D. (2013). Knowledge creating and sharing corporate culture framework. Procedia-Social and Behavioral Sciences, 74, 251-260.

Wang, S., \& Noe, R. A. (2010). Knowledge sharing: A review and directions for future research. Human Resource Management Review, 20(20), 115-131 